\title{
NOVEL FRACTALS OF HUTCHINSON BARNSLEY OPERATOR IN HAUSDORFF $G$-METRIC SPACES
}

\author{
SUDESH KUMARI ${ }^{\dagger}$ AND RENU CHUGH
}

\author{
Date of Receiving : $\quad 23.12 .2019$ \\ Date of Revision : $\quad 05.03 .2020$ \\ Date of Acceptance : 12.05 .2020
}

\begin{abstract}
In this article, we construct fractals (attractors) with the help of iterated function systems (IFS) which is a finite family of contraction mappings defined on Hausdorff generalized metric $(G$ - metric) space. In this proposed space, we prove some results to obtain attractors for Hutchinson Barnsley (HB) operator which satisfy different contractive conditions. We derive Collage theorem which is used to find out suitable iterated function systems (IFS) for constructing fractals. Also, we have presented some practical examples of classical fractals to validate our results. One can further generalize and extend our results and construct fractals or multifractals by using more general contractive conditions.
\end{abstract}

\section{Introduction}

In today's scenario of research, fractal theory has been applied in many diverse branches of science due to its extensive applications. The term fractal was firstly used by Benoit Mandelbrot in his book "The Fractal Geometry of Nature" (1982) and defined it as "rough or fragmented geometric shapes that can be split into congruent pieces, each of which is a reduced size copy of the origin one". Various techniques like Iterated Function Systems, Escape Time Fractals, Strange Attractors and Random Fractals have been adopted to generate Fractals.

This paper is devoted to Iterated Function Systems (IFS) technique to generate fractals. Hutchinson [20] defined iterated function systems (IFS) and Barnsley [9] enriched the theory of IFS and named it as Hutchinson Barnsley (HB) theory. Hutchinson introduced IFS as a finite collection of contractive self mappings and defined HB operator on hyperspace of nonempty compact sets. He showed that the unique fixed point of HB operator is a fractal (attractor). Thus, fractals can be constructed by using fixed point theory. For years, IFS has become emerging technique for researchers

2010 Mathematics Subject Classification. 28A80, 47H09, 47H10.

Key words and phrases. Fractals, iterated function systems, contraction, Hutchinson Barnsley operator, Hausdorff $G$ - metric spaces.

Communicated by. Zead Mustafa

${ }^{\dagger}$ Corresponding author. 